\title{
Modeling And Simulation Of Polar Coordinates Seeker Control System Based On Full-Map Plane
}

\author{
Yufu Guo, Ping Zhang \\ School of Automation Science and Electric Engineering, BUAA \\ Beijing, China \\ e-mail: guoyufu2011@163.com
}

\begin{abstract}
A control system of polar coordinates seeker has been modeled, utilizing an angle instruction solution based on full-map plane and nonlinear PID (NLPID) controller based on tracking differentiator (TD) and extended state observer (ESO). The jump of instruction is solved by rolling the fullmap coordinate system, accomplishing the optimization of the many-valued instruction of polar coordinates seeker. Then the system uses TD to filter the optimized instruction and to construct its differential signal, and uses differential signal to predict it by linear extrapolation. The two servo channels have same structure, both using optimal control synthesis function of discrete-time system - fhan() function to construct NLPID to drive the electromotor. In the angular velocity feed-back channel, system uses ESO to filter the signal and to estimate the disturbance and compensate it. Simulation results for sight stabilization and target tracking shows that the system's decoupling coefficient and dislocation angle when tracking complicated trace are both small.
\end{abstract}

Keywords- polar coordinates; seeker; full-map plane; NLPID

\section{PREFACE}

Polar coordinates seeker is an important part of new generation of close combat air-to-air missile due to its compact structure and ability of high off-boresight launch .Institutes home and abroad have made research about polar coordinates seeker mainly in following aspects [1]: optical-mechanical structure, principle of target tracking and stabilizing, problem of tracking in zenith blind zone and strategy for it, servo control method. Present domestic tracking principles of polar coordinates seeker are nearly all using angle increment instruction calculated from target's position on detector and frames' deflection angles. Specifically there are traditional method according to two different rotation ways from line of sight to line of target - line from origin of coordinates to target, which needs logical judgment to optimize instruction [2,3]; and method based on Paden-Kahan subproblem, which applies the minimum angle increment criteria to optimize the inverse kinematics problem [4]. Strategies for target tracking in zenith zone under above instruction solution mainly include instruction prediction and varying parameters in different regions $[1,3]$. Servo controllers are mainly designed on the basis of frequency domain theory [1].

The modeling in this article aims at two aspects: target tracking principle and servo control solution. The target tracking principle includes instruction calculation and optimization. Different from past calculating angle increment instruction, this principle uses angle instruction calculated by full-map plane and optimized it by rotating coordinate system of full-map plane. The servo applies nonlinear PID (NLPID) controller based on tracking differentiator(TD), and utilizes extended state observers(ESO) in angular velocity feedback loop [5], accomplishing adaptive control. TD can make dynamic planning and prediction of angular instruction, while angle increment instruction is not fit for dynamic planning because optical axis itself is in great movement. This embodies angle instruction's superiority to angle increment instruction. The control system was simulated in Simulink environment of Matlab. The results displayed that the system's decoupling coefficient is $2.89 \%$, and the dislocation angle during complex target tracking was under $1.4^{\circ}$

\section{REFERENCE FRAMES}

The reference frames used in this system model have the same origin $\mathrm{O}$ - the intersection of the framework's shafts. There are base-fixed reference frame $F_{B}$, and the base carried local earth reference frame $F_{I}$ with its origin orientation same with $F_{B}$ and keeps unchanged relative to inertial space; the outer gimbal-fixed reference frame $\mathrm{F}_{\mathrm{O}}$, obtained from $\mathrm{F}_{\mathrm{B}}$ by rotation of $\theta_{o x}$ i.e. roll angle; the inner gimbal-fixed reference frame $\mathrm{F}_{\mathrm{P}}$, obtained from $\mathrm{F}_{\mathrm{O}}$ by rotation of $\theta_{p y}$ i.e. pitch angle; full-map plane $\mathrm{x}-\mathrm{y}$, obtained by spreading image sphere-sphere ahead of seeker with $\mathrm{O}$ as origin and $f$ as radius; instantaneous image plane $\mathrm{dx}-\mathrm{dy}$ which expresses the location of target on the detector as is also indicated in Fig. 1. The $f$ is the focal length of the detector, which is also equal to $O Q$.

There is equation turning $\left(x_{b}, y_{b}, z_{b}\right)$ in $\mathrm{F}_{\mathrm{B}}$ to $(x, y)$ in fullmap plane:

$$
\left\{\begin{array}{c}
\alpha=\arctan \frac{-z_{b}}{y_{b}}+\frac{1-\operatorname{sign}\left(y_{b}\right)}{2} \cdot \pi \\
\beta=\operatorname{arcos} \frac{x_{b}}{\sqrt{x_{b}^{2}+y_{b}^{2}+z_{b}^{2}}} \\
x=f \beta \cos \alpha \\
y=f \beta \sin \alpha
\end{array}\right.
$$

For a random point $\mathrm{P}$ in $\mathrm{F}_{\mathrm{B}}$, its corresponding $\alpha, \beta$ are showed in Fig. 2. 


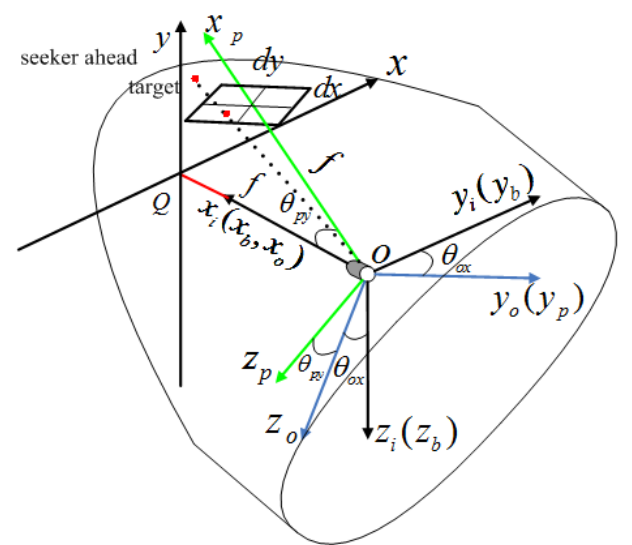

Figure 1. Reference frames: FI, FB, FO, FP, x-y, dx-dy

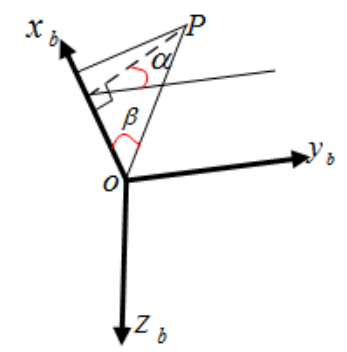

Figure 2. Indication of $\alpha, \beta$

\section{CONTROL SYSTEM'S STRUCTURE AND ALGORITHM}

\section{A. Control Configuration}

The system adopts angle tracking method. First, the detector gets the coordinates of target on instantaneous image plane- - $\left(d_{x}, d_{y}\right)$, so the target's coordinates in $\mathrm{F}_{\mathrm{P}}$ is $(f$, $\left.d_{x},-d_{y}\right)$, which is to be converted to coordinates in $\mathrm{F}_{\mathrm{B}}$ according to $\theta_{o x}, \theta_{p y}$. Then use Equation (1) to get target's coordinates in full-map plane-— $(x, y)$. Next utilize $(x, y)$ to calculate and optimize angle instruction.

\section{B. Control System's Structure}

After instruction's calculation and optimization in fullmap plane, system utilizes TD to filter and predict it, and to construct its differential signal at the same time, which equivalently makes dynamic planning for it. Predicted instruction and its differential signal respectively minus feedbacks of framework's angle and angular velocity are their deviation signals. Then controller utilize optimal control synthesis function of discrete-time system - - fhan() function to combine angle deviation, angular velocity deviation, integral of angle deviation in a nonlinear way to obtain control signal. In angle velocity feedback loop, system utilizes ESO to estimate high-order terms and disturbing torque in the dynamic equation of framework's motor and compensate them. System's control destination is to obtain a low decoupling coefficient and low dislocation angle.

The control system's structure is indicated in Fig. 3. $(x, y)$ is target's coordinates in full-map plane, $\theta_{o x c}, \theta_{p y c}$ are angle instruction of outer and inner gimbals i.e. roll instruction and pitch instruction. $\hat{\theta}_{o x c}, \hat{\theta}_{p y c}$ and $\dot{\hat{\theta}}_{o x c}, \dot{\hat{\theta}}_{p y c}$ are respectively predictions and differentials of $\theta_{o x c}, \theta_{p y c}$. On feedback loops of gimbals' angular velocities, velocities $\dot{\theta}_{o x}$ and $\dot{\theta}_{p y}$ are respectively inputted to ESO, ESO outputs velocities' filtered values $--\hat{\dot{\theta}}_{o x}, \hat{\dot{\theta}}_{p y}-$ and estimated values of controlled objects' disturbance, i.e. extended state. The extended states are used to compensate controlled objects' disturbance.

\section{Instruction Calculation And Optimization [6]}

\section{1) Primary instruction equation}

According to geometrical structure shown in Fig. 1, the instruction can be calculated by Equation (3).

$$
\left\{\begin{array}{c}
\theta_{o x c}=\operatorname{sign}(x \cdot y) \cdot \arctan |x / y| \\
\theta_{p y c}=\operatorname{sign}(y) \cdot \sqrt{x^{2}+y^{2}} / f
\end{array}\right.
$$

$\theta_{o x c}, \theta_{p y c}$ in Equation (3) take radian as unit.

2) Instruction jump and optimization

Utilizing Equation (3), there will be jump of instruction when target's image point cross $\mathrm{X}$-axis of full-map plane as is indicated in Fig. 4.

$\left(x_{0}, y_{0}\right)$ and $(x, y)$ are respectively coordinates of the last target's image point on full-map plane and the current one. Here $\alpha, \gamma$ are respectively last point's and current point's angular instructions of outer gimbal. Obviously, $\beta$ is more appropriate as current point's instruction. And $\beta$ can be obtained by following method: rotate $\mathrm{x}-\mathrm{y}$ clockwise- - as is the way image point crosses the $\mathrm{x}$-axis—— to $x^{\prime}-y^{\prime}$, then current point's coordinates in $x^{\prime}-y^{\prime}$ is : $\left\{\begin{array}{c}x^{\prime}=-y \\ y^{\prime}=x\end{array}\right.$, and $\beta=\arctan \left|x^{\prime} / y^{\prime}\right|+\pi / 2$.
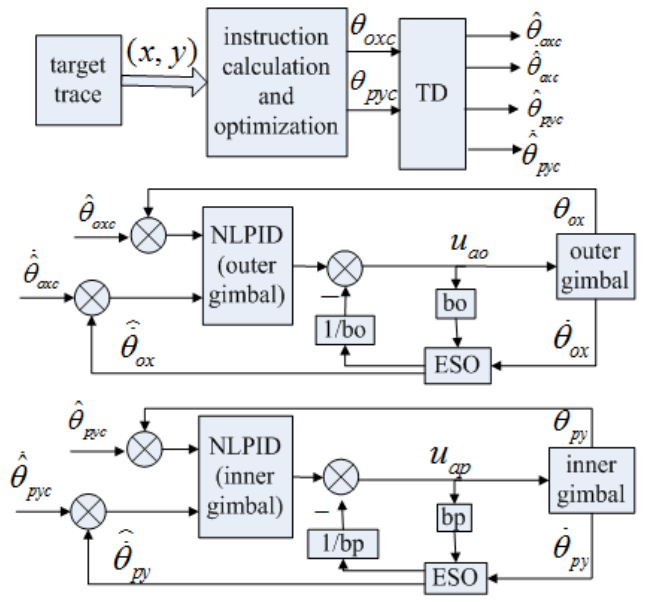

Figure 3. Structure of the control system 


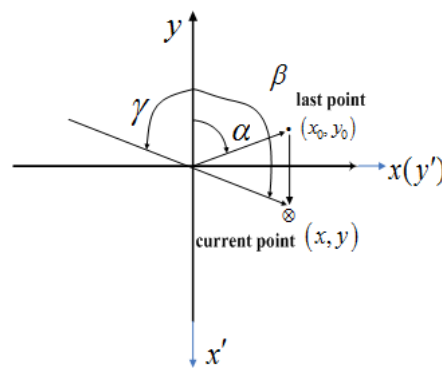

Figure 4. Indication of instruction jump

Above all, the way of instruction optimization can be summarized as the following: when target's image point on full-map plane crosses $x$-axis, rotate $x-y$ the way image point cross $x$-axis by $\pm 90^{\circ}$ to $x^{\prime}-y^{\prime}$. Then obtain image point's new coordinates $\left(x^{\prime}, y^{\prime}\right)$ and substitute $\left(x^{\prime}, y^{\prime}\right)$ into Equation (3), the calculated $\theta_{\text {oxc }}$ plus with clockwise rotation times of $\pi / 2$ is the final roll instruction and the calculated $\theta_{p y c}$ is the final pitch instruction.

\section{3) Rotation criteria and final instruction equation}

The following Table I and Table II respectively list criteria to rotate $\mathrm{x}-\mathrm{y}$ clockwise and anticlockwise.

Taking flg as clockwise rotation time and take anticlockwise rotation as negative, the final instruction is calculated as Equation (4). The $\left(x^{\prime}, y^{\prime}\right)$ is the coordinates of current target's image point in rotated full-map plane reference frame- $-x^{\prime}-y^{\prime}$.

$$
\left\{\begin{array}{c}
\theta_{o x c}=\operatorname{sign}(x \cdot y) \cdot \arctan |x / y|+f \lg \cdot \pi / 2 \\
\theta_{p y c}=\operatorname{sign}(y) \cdot \sqrt{x^{2}+y^{2}} / f
\end{array}\right.
$$

TABLE I. CRITERIA TO ROTATE X-Y CLOCKWISE

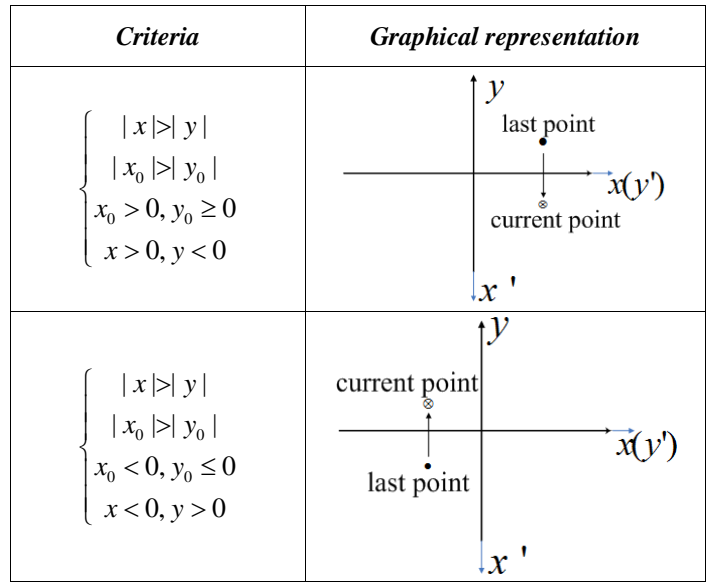

TABLE II. CRITERIA TO ROTATE X-Y ANTICLOCKWISE

\begin{tabular}{|l|l|}
\hline Criteria & Graphical representation \\
\hline
\end{tabular}

\begin{tabular}{|c|c|c|}
\hline Criteria & Graphical re & epresentation \\
\hline $\begin{array}{c}|x|>|y| \\
\left|x_{0}\right|>\left|y_{0}\right| \\
x_{0}>0, y_{0}<0 \\
x>0, y \geq 0\end{array}$ & $y^{\prime}$ & $\begin{array}{l}y\left(x^{\prime}\right) \\
\text { current point } \\
\stackrel{\text { last point }}{\longrightarrow} x\end{array}$ \\
\hline $\begin{array}{c}|x|>|y| \\
\left|x_{0}\right|>\left|y_{0}\right| \\
x_{0}<0, y_{0}>0 \\
x<0, y \leq 0\end{array}$ & current point & $y\left(x^{\prime}\right)$ \\
\hline
\end{tabular}

After optimization, the instruction won't jump when target's image point crosses $\mathrm{X}$-axis.

D. NLPID $[5,7,8]$

\section{1) PID's shortcomings}

Utilizing PID controller, we don't need to know controlled object's accurate math model, and just use the deviation before object's output and input to construct control variable. So PID controller has been widely used. But PID controller has the following shortcomings:

- Control variable's jump may cause overshoot.

- Deviation's differential is not easy to implement.

- Linear combination of deviation's proportion, differential, integral sometimes can't reach good effect.

NLPID based on TD and ESO can effectively solve above problem.

2) $T D$

TD's principle can be seen in Reference [4], and the main work to construct a TD is to choose a fine acceleration function. TD in this system utilizes arctangent function shown in Equation (5) as acceleration function [9].

$$
g\left(x_{1}, x_{2}\right)=a_{1} \arctan \left(f_{1} x_{1}\right)+a_{2} \arctan \left(f_{2} x_{2}\right)
$$

$a_{1}, a_{2}, f_{1}, f_{2}$ are positive constants. The TD's dynamic is expressed by Equation (6).

$$
\left\{\begin{array}{c}
\dot{x}_{1}=x_{2} \\
\dot{x}_{2}=-R^{2}\left\{a_{1} \arctan \left[f_{1}\left(x_{1}-v\right)\right]+a_{2} \arctan \left(f_{2} x_{2} / R\right)\right\}
\end{array}\right.
$$

In Equation (6) $v$ is TD's input i.e. angle instruction. $x_{1}$ is tracking signal of $v$, and $x_{2}$ is differential of $x_{1}$ which acts as approximation of $v$ 's differential. Denoting

$$
g(e)=R^{2}\left\{a_{1} \arctan \left[f_{1}\left(x_{1}-v\right)\right]+a_{2} \arctan \left(f_{2} x_{2} / R\right)\right\} \quad \text { while }
$$

$e=x_{1}-v$ is the angular tracking deviation, Equation (7) can be derived from Equation (6) by discretization. 


$$
\left\{\begin{array}{c}
x_{1}(k+1)=x_{1}(k)+h x_{2}(k) \\
x_{2}(k+1)=x_{2}(k)+h[-g(e)]
\end{array}\right.
$$

$h$ is discrete system's period. Utilizing TD's output $x_{1}, x_{2}$ to make linear extrapolation by $p$ steps gets the prediction of instruction, as is expressed by Equation (8).

$$
x_{f c}=x_{1}+p \cdot h \cdot x_{2}
$$

$x_{f c}$ is the prediction of angular instruction. This system has used four TD with structure shown in Fig. 5. TD3 and TD4 are used to filter differentials outputted by TD1 and TD2, making gimbal's angular velocity instruction more smooth.

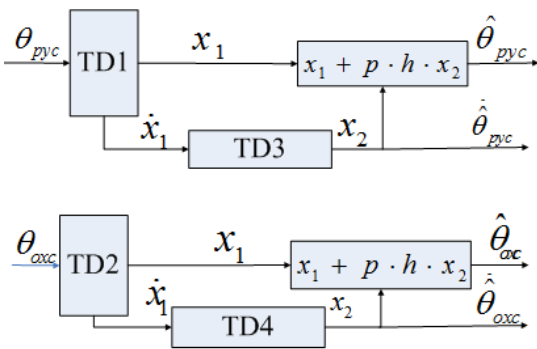

Figure 5. Instruction filter and prediction and dynamic planning

3) Nonlinear combination in NLPID-fhan() function

NLPID in Fig. 3 utilizes fhan() function to combination deviation's proportion, integral and differential to construct control variable. Nonlinear fhan() and control variable is expressed in Equation (9) [7]. $e_{1}, e_{2}$ are respectively angular deviation and angular velocity deviation i.e. the two inputs of NLPID shown in Fig. 3. $e_{0}=\int e_{1}$ is angular deviation's integral.

$$
\left\{\begin{array}{c}
h_{1}=h \cdot r_{1} \\
\delta=h_{1} \cdot r \\
\delta_{1}=h_{1} \cdot \delta \\
y=e_{1}+c \cdot h_{1} \cdot e_{2} \\
g=\left\{\begin{array}{c}
e_{2}+\operatorname{sign}(y) \cdot \frac{\sqrt{8 r \cdot \mid+\delta^{2}}}{2}, \delta \\
e_{2}+\frac{y}{h_{1}},|y|<\delta_{1}
\end{array} \mid y \delta_{1}\right. \\
\operatorname{sat}(g, \delta)=\left\{\begin{array}{c}
\operatorname{sign}(g),|g| \geq \delta \\
\frac{g}{\delta},|x|<\delta \\
\text { fhan }\left(e_{1}, e_{2}, r, h_{1}\right)=-r \cdot \operatorname{sat}(g, \delta) \\
u=k_{0} e_{0}+
\end{array}\right.
\end{array}\right.
$$

\section{4) $\mathrm{ESO}$}

ESO can estimate uncertain part of controlled object's dynamic equation, and be used in controller to compensate the error of model and unknown outer disturbance [10]. So it's fit for seeker having strong dynamic coupling uncertain disturbance moment.

In this system, ESO is used in gimbals' angular velocity feedback loop, taking angular velocity as input and outing its filtered value and extended state- - estimation of uncertain part of controlled object. The extended state is used for compensation as is shown in Fig. 3.

Considering of error of model and disturbance, gimbal motor's dynamic can be expressed by Equation (10).

$$
\dot{x}=w(x, t)+b u
$$

$x$ is motors angular velocity. $w(x, t)$ is decided by back electromotive force, high derivative of $x$, coupling moment and disturbance moment etc. and it's hard to model accurately. System's state is one-dimensional, plus extended state it'll be two-dimensional. Utilize arctangent function to construct ESO as expressed by Equation (11).

$$
\left\{\begin{array}{c}
\dot{z}_{1}=z_{2}-\beta_{01} \cdot e+b \cdot u \\
\dot{z}_{2}=-\beta_{02} \cdot \arctan (f \cdot e)
\end{array}\right.
$$

$$
e=z_{1}-x, \quad \beta_{01}, \beta_{02}, f>0, z_{1}, z_{2} \text { respectively track } x,
$$
$w(x, t)$. The compensation should achieve: $\dot{x}=w(x, t)+b\left(u-k z_{2}\right) \approx w(x, t)+b(u-k w)=b u \quad, \quad$ so compensation coefficient $k=1 / b$, as is indicated in Fig. 3. Equation (12) is derived by discretization of Equation (11).

$$
\left\{\begin{array}{c}
z_{1}(k+1)=z_{1}(k)+h\left[z_{2}(k)-\beta_{01} \cdot e(k)+b \cdot u(k)\right] \\
z_{2}(k+1)=z_{2}(k)+h\left\{-\beta_{02} \cdot \arctan [f \cdot e(k)]\right\}
\end{array}\right.
$$

\section{SIMULATION AND ANALYSIS}

\section{A. Simulation of Sight Stabilization}

Target keep static on $\mathrm{x}$-axis reference frame $\mathrm{F}_{\mathrm{I}}$, and base has triaxial angular velocity of sinusoid with amplitude of $3^{\circ} / \mathrm{s}$ and frequency of $1 \mathrm{~Hz}$. The off-boresight can be seen in Fig. 6 and dislocation angle in Fig. 7.

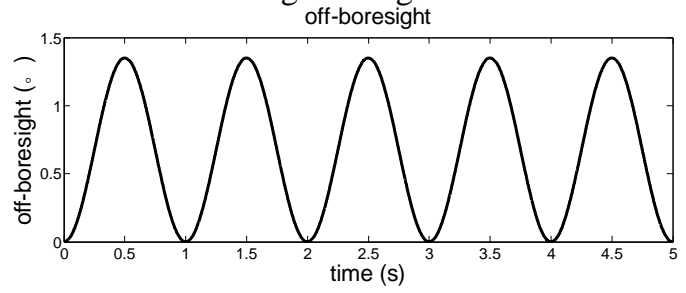

Figure 6. Off-boresight $\left(^{\circ}\right)$ in sight stabilization simulation 


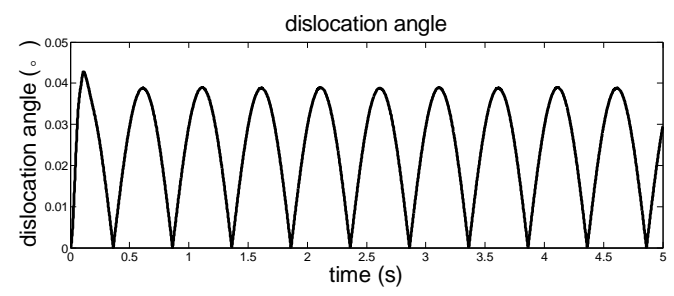

Figure 7. Dislocation angle $\left(^{\circ}\right)$ in sight stabilization simulation

Using off-boresight's amplitude $1.35^{\circ}$ to divide dislocation angle's amplitude $0.039^{\circ}$ gets system's decoupling coefficient, which equals $2.89 \%$.

\section{B. Simulation Of Target Tracking With Base's Disturbance}

In this case, base has triaxial angular velocity of sinusoid with amplitude of $3^{\circ} / \mathrm{s}$ and frequency of $1 \mathrm{~Hz}$. Meanwhile target maneuvers in $\mathrm{F}_{\mathrm{I}}$. Figure 8 shows target's and optical axis's traces on full-map plane. Figure 9 shows targets trace in detector's instantaneous image plane dx-dy. Figure 10 shows the dislocation angle. Figure 11 shows gimbal's angular instruction, including original and predicted, and real angular response.

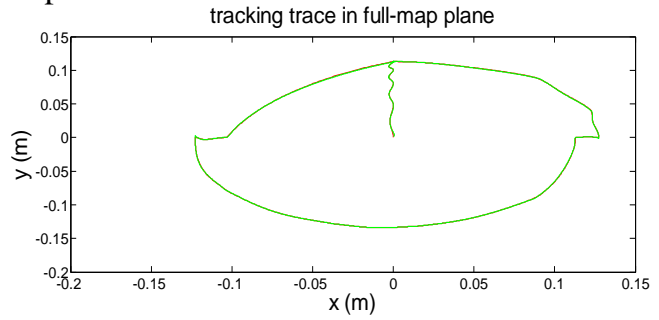

Figure 8. Target's and optical axis's trace on full-map plane(target's trace by red line and optical axis's by green line)

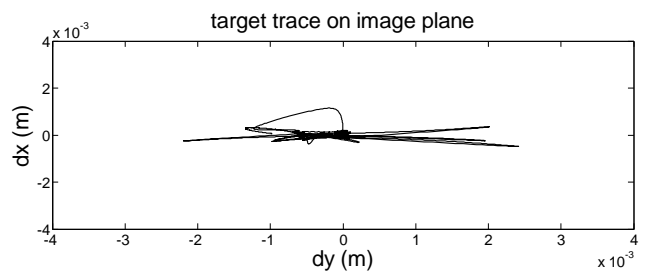

Figure 9. Target's trace on detector's image plane

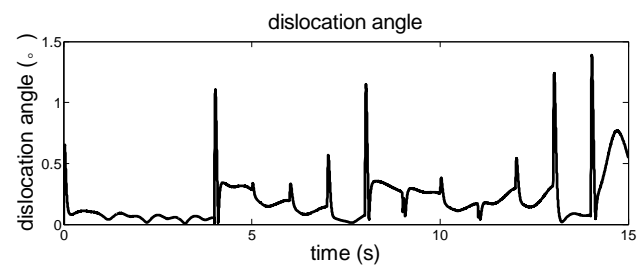

Figure 10. Dislocation angle $\left({ }^{\circ}\right)$

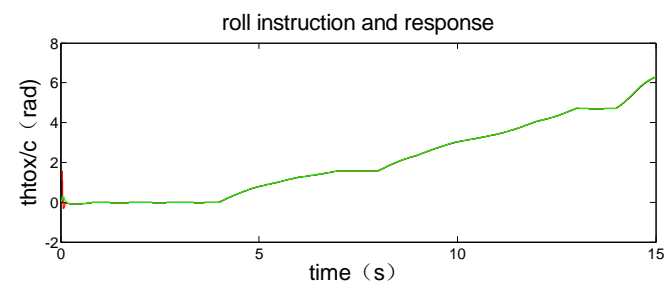

(a)

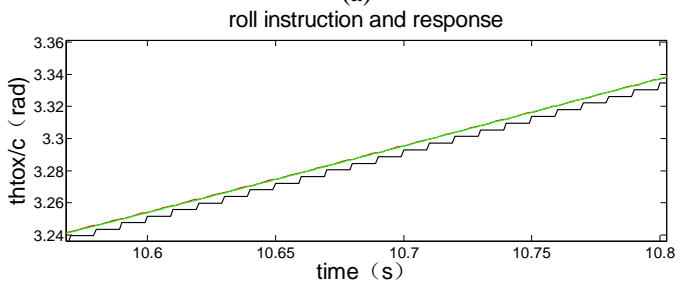

(b)

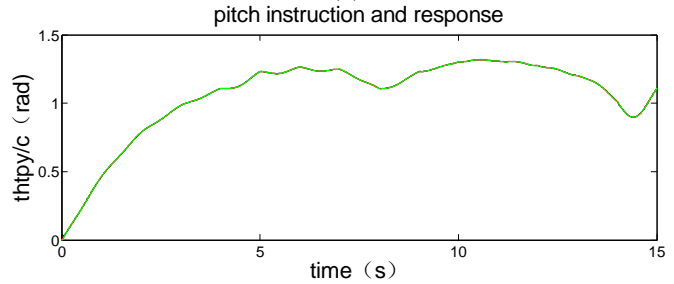

(c)

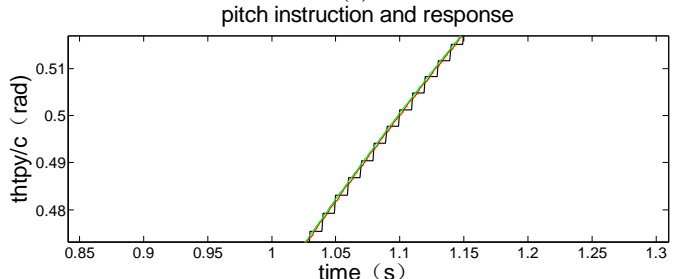

(d)

Figure 11. (a)(b) are roll instruction and response's global/partial data(original instruction by black line, the predicted by red line, the response by green line); (c)(d) pitch instruction and response's global/partial data(original instruction by black line, the predicted by red line, the response by green line)

Fig. 8, Fig. 9 and Fig. 10 show that the optical axis tracks target very well, the dislocation angle is under $1.4^{\circ}$. Fig. 11 (b),(d) show that TD functions effectively in filtering and predicting instruction signal, and response track the predicted instruction efficiently, proving NLPID and ESO are fit for this system.

\section{CONCLUSION AND PROSPECT}

The optimization of instruction by rotating reference frame on full-map plane has solved instruction's jump, offering an angular instruction solution which is fitter for dynamic planning and prediction than angular increment instruction. Controller utilizing NLPID based on TD and ESO is appropriate for seeker's servo. In the future corresponding strategy for target tracking in zenith blind zone based on instruction solution of this paper is needed.

\section{REFERENCES}

[1] Jiang Huhai. "Research on Strategy for Target Tracking in Zenith Zone of Roll-Pitch Seeker"[D]. Changchun: Changchun Institute of 
Optics, Fine Mechanics and Physics, Chinese Academy of Sciences, 2012.

[2] WANG Zhi-wei, Qi Zai-kang and WANG Jiang. "Tracking principle for roll-pitch seeker"[J]. Infrared and Laser Engineering, 2008, vol.37,No.2, pp.274-277.

[3] LIN De-fu, WANG Zhi-wei and WANG Jiang. "Singularity Anaysis of Roll-pitch Seeker and Its Control Strategy"[J]. Transactions of Beijing Institute of Technology, 2010,vol.30,No.11, pp.1265-1269.

[4] Zhu Ming-chao, JIA Hong-guang. "Solution of angle increments for roll-pitch seekers based on Paden-Kahan sub-problems"[J]. Optics and Precision Engineering, 2011, vol.19,No.8, pp.1838-1844.

[5] Han Jing-qing. "A New Kind of Controller-NLPID"[J]. Control and Decision, 1994, vol.9, No.6, pp.401-406;
[6] Zhang Ping, Dong Xiao-meng, Fu Kui-sheng and Deng Fang-yi. "Modeling and Control of Airborne/Missile-borne Vision-Guidances Stabilized Platform'[M]. Beijing: National Defense Industry Press, 201, pp.92-94.

[7] Han Jing-qing. "Auto Disturbances Rejection Control Technique"[J]. Frontier Science, 2007, No.1, pp.24-31.

[8] Han Jing-qing. "From PID to Active Disturbance Rejection Control'[J]. IEEE TRANSACTIONS ON INDUSTRIAL ELECTRONICS, 2009, vol.56, No.3, pp.900-906.

[9] Dong Xiao-meng and Zhang Ping. "Design and phase plane analysis of an arctangent-based tracking differentiator"'[J]. Control Theory \& Applications, 2010, vol.27, No.4, pp.533-537.

[10] Han Jing-Qing. "A kind of extended state observer for uncertain objects"[J]. Control and Decision, 1995, vol.10, No.1, pp.85-88. 